\title{
$\eta^{\prime} \rightarrow \gamma \gamma$ and the topological susceptibility
}

\author{
G.M. Shore ${ }^{\mathrm{a} * \dagger}$ \\ ${ }^{\mathrm{a}} \mathrm{TH}$ Division, CERN \\ CH1211 Geneva 23, Switzerland.
}

The radiative decays $\eta^{\prime}(\eta) \rightarrow \gamma \gamma$ are discussed. The modifications of the conventional PCAC formulae due to the gluonic contribution to the flavour singlet axial anomaly are given. The decay constants satisfy a modified Dashen formula which generalises the Witten-Veneziano formula for the mass of the $\eta^{\prime}$. It is shown how the topological susceptibility in QCD with massive, dynamical quarks may be extracted from measurements of $\eta^{\prime}(\eta) \rightarrow \gamma \gamma$.

\section{The Result}

Radiative decays of the $\eta^{\prime}$ are currently attracting renewed interest. (See [2] for references.) In this talk we summarise the special features that arise due to the gluonic contribution to the $U_{A}(1)$ anomaly when PCAC methods (including chiral Lagrangians) are used in the flavour singlet channel. In [1], we presented an analysis of $\eta^{\prime} \rightarrow \gamma \gamma$ decay in the chiral limit of QCD, taking into account the gluonic anomaly and the associated anomalous scaling implied by the renormalisation group. Here, we summarise the results of a recent new analysis 20xtending this to QCD with massive quarks, incorporating $\eta-\eta^{\prime}$ mixing. In particular, we show how a combination of the radiative decay formula and a generalisation of the Witten-Veneziano mass formula for the $\eta^{\prime}$ may be used to measure the gluon topological susceptibility $\chi(0)$ in full QCD with massive quarks.

Our main result is summarised in the formulae:

$f^{a \alpha} g_{\eta^{\alpha} \gamma \gamma}+2 n_{f} A g_{G \gamma \gamma} \delta_{a 0}=a_{\mathrm{em}}^{a} \frac{\alpha}{\pi}$

which describes the radiative decays, and

$$
\begin{aligned}
& f^{a \alpha}\left(m^{2}\right)_{\alpha \beta} f^{T \beta b}=\left(2 n_{f}\right)^{2} A \delta_{a 0} \delta_{b 0} \\
& -2 d_{a b c} \operatorname{tr} T^{c}\left(\begin{array}{ccc}
m_{u}\langle\bar{u} u\rangle & 0 & 0 \\
0 & m_{d}\langle\bar{d} d\rangle & 0 \\
0 & 0 & m_{s}\langle\bar{s} s\rangle
\end{array}\right)
\end{aligned}
$$

*Permanent address: Dept. of Physics, University of Wales Swansea, Singleton Park, Swansea, SA2 8PP, U.K. ${ }^{\dagger}$ CERN-TH/99-238, SWAT/99-235, hep-ph/9908273. To appear in the Proceedings, QCD99 Montpellier which defines the decay constants appearing in (1) through a modification of Dashen's formula to include the gluon contribution to the $U_{A}(1)$ anomaly.

In these formulae, $\eta^{\alpha}$ denotes the neutral pseudoscalars $\pi^{0}, \eta, \eta^{\prime}$. The (diagonal) mass matrix is $\left(m^{2}\right)_{\alpha \beta}$ and $g_{\eta^{\alpha} \gamma \gamma}$ is the appropriate coupling, defined as usual from the decay amplitude by $\left\langle\gamma \gamma \mid \eta^{\alpha}\right\rangle=-i g_{\eta^{\alpha} \gamma \gamma} \epsilon_{\lambda \rho \alpha \beta} p_{1}^{\alpha} p_{2}^{\beta} \epsilon^{\lambda}\left(p_{1}\right) \epsilon^{\rho}\left(p_{2}\right)$. The constant $a_{\mathrm{em}}^{a}$ is the coefficient of the electromagnetic contribution to the axial current anomaly:

$\partial^{\mu} J_{\mu 5}^{a}=d_{a c b} m^{c} \phi_{5}^{b}+2 n_{f} \delta_{a 0} Q+a_{\mathrm{em}}^{a} \frac{\alpha}{8 \pi} F^{\mu \nu} \tilde{F}_{\mu \nu}(3)$

Here, $J_{\mu 5}^{a}$ is the axial current, $\phi_{5}^{a}=\bar{q} \gamma_{5} T^{a} q$ is the quark pseudoscalar and $Q=\frac{\alpha_{s}}{8 \pi} \operatorname{tr} G^{\mu \nu} \tilde{G}_{\mu \nu}$ is the gluon topological charge. $m^{a}$ are the quark masses (see eq(11)). $a=0,3,8$ is the flavour index, $T^{3,8}$ are $S U(3)$ generators and $T^{0}=\mathbf{1}$. The $d$-symbols are defined by $\left\{T^{a}, T^{b}\right\}=d_{a b c} T^{c}$.

The decay constants $f^{a \alpha}$ in (1) are defined by the relation (2). In general they are not the couplings of the pseudoscalar mesons to the axial current[1]. In the flavour singlet sector, such a definition would give a RG non-invariant decay constant which would not coincide with the quantities arising in the correct decay formula (1). In contrast, all the quantities in the formulae (1),(2) are separately RG invariant [2], 3. The proof is not immediately obvious, and depends on the RGEs for the various Green functions and vertices defining the terms in (1),(2) being evaluated on-shell or at zero-momentum.

In practice, since flavour $S U(2)$ symmetry is al- 
most exact, the relations for $\pi^{0}$ decouple and are simply the standard ones with $f^{3 \pi}$ identified as $f_{\pi}$ (see eqs $(24),(27)$ ). In the octet-singlet sector, however, there is mixing and the decay constants form a $2 \times 2$ matrix:

$f^{a \alpha}=\left(\begin{array}{ll}f^{0 \eta^{\prime}} & f^{0 \eta} \\ f^{8 \eta^{\prime}} & f^{8 \eta}\end{array}\right)$

The four components are independent. In particular, for broken $S U(3)$, there is no reason to express $f^{a \alpha}$ as a diagonal matrix times an orthogonal $\eta-\eta^{\prime}$ mixing matrix, which would give just three parameters. Several convenient parametrisations may be made, e.g. involving two constants and two mixing angles, but this does not seem to reflect any special dynamics.

The novelty of our results of course lies in the extra terms arising in (1) and (2) due to the gluonic contribution to the $U_{A}(1)$ anomaly. The coefficient $A$ is the non-perturbative number which specifies the topological susceptibility in full QCD with massive dynamical quarks. The topological susceptibility is defined as

$\chi(0)=\int d^{4} x i\langle 0|T Q(x) Q(0)| 0\rangle$

The anomalous chiral Ward identities determine its dependence on the quark masses and condensates up to an undetermined parameter, viz.

$\chi(0)=-A\left(1-A \sum_{q} \frac{1}{m_{q}\langle\bar{q} q\rangle}\right)^{-1}$

Notice how this satisfies the well-known result that $\chi(0)$ vanishes if any quark mass is set to zero.

The modified flavour singlet Dashen formula is in fact a generalisation of the Witten-Veneziano mass formula for the $\eta^{\prime}$. Here, however, we do not impose the leading order in $1 / N_{c}$ approximation that produces the Witten-Veneziano formula. Recall that this states

$m_{\eta^{\prime}}^{2}+m_{\eta}^{2}-2 m_{K}^{2}=-\left.\frac{6}{f_{\pi}^{2}} \chi(0)\right|_{\mathrm{YM}}$

To recover (7) from our result (see the first of eqs $(9))$ the condensate $m_{s}\langle\bar{s} s\rangle$ is replaced by the term proportional to $f_{\pi}^{2} m_{K}^{2}$ using a standard
Dashen equation, and the singlet decay constants are set to $\sqrt{2 n_{f}} f_{\pi}$. The identification of the large $N_{c}$ limit of the coefficient $A$ with the non-zero topological susceptibility of pure Yang-Mills theory follows from large $N_{c}$ counting rules and is explained in ref[2].

The final element in (1) is the extra 'coupling' $g_{G \gamma \gamma}$ in the flavour singlet decay formula, which arises because even in the chiral limit the $\eta^{\prime}$ is not a Goldstone boson because of the gluonic $U_{A}(1)$ anomaly. A priori, this is not a physical coupling, although (suitably normalised) it could be modelled as the coupling of the lightest predominantly glueball state mixing with $\eta^{\prime}$. However, this interpretation would probably stretch the basic dynamical assumptions underlying (1) too far, and is not necessary either in deriving or interpreting the formula. In fact, the $g_{G \gamma \gamma}$ term arises simply because in addition to the electromagnetic anomaly the divergence of the axial current contains both the quark pseudoscalar $\phi_{5}^{a}$ and the gluonic anomaly $Q$. Diagonalising the propagator matrix for these operators isolates the $\eta$ and $\eta^{\prime}$ poles, whose couplings to $\gamma \gamma$ give the usual terms $g_{\eta \gamma \gamma}$ and $g_{\eta^{\prime} \gamma \gamma}$. However, the remaining operator (which we call $G$ ) also couples to $\gamma \gamma$ and therefore also contributes to the decay formula, whether or not we assume that its propagator is dominated by a 'glueball' pole.

Of course, the presence of the coupling $g_{G \gamma \gamma}$ in (1) appears to remove any predictivity from the $\eta^{\prime} \rightarrow \gamma \gamma$ decay formula. In a strict sense this is true, but we shall argue that it may nevertheless be a good dynamical approximation to assume $g_{G \gamma \gamma}$ is small compared to $g_{\eta^{\prime} \gamma \gamma}$. In this case, we can combine eqs (1) and (2) to give a measurement of the non-perturbative coefficient $A$ in $\chi(0)$. To see this, assume $m_{u}=m_{d} \simeq 0$. The terms in (1), (2) involving the pion then decouple leaving the following five equations, in which we assume the physical quantities $m_{\eta}, m_{\eta^{\prime}}, g_{\eta \gamma \gamma}$ and $g_{\eta^{\prime} \gamma \gamma}$ are all known and we neglect $g_{G \gamma \gamma}$. The decay equations are:

$$
\begin{gathered}
f^{0 \eta^{\prime}} g_{\eta^{\prime} \gamma \gamma}+f^{0 \eta} g_{\eta \gamma \gamma}+6 A g_{G \gamma \gamma}=a_{\mathrm{em}}^{0} \frac{\alpha}{\pi} \\
f^{8 \eta} g_{\eta \gamma \gamma}+f^{8 \eta^{\prime}} g_{\eta^{\prime} \gamma \gamma}=a_{\mathrm{em}}^{8} \frac{\alpha}{\pi}
\end{gathered}
$$

where $a_{\mathrm{em}}^{0}=\frac{4}{3} N_{c}$ and $a_{\mathrm{em}}^{8}=\frac{1}{3 \sqrt{3}} N_{c}$, and the 
Dashen equations are:

$$
\begin{gathered}
\left(f^{0 \eta^{\prime}}\right)^{2} m_{\eta^{\prime}}^{2}+\left(f^{0 \eta}\right)^{2} m_{\eta}^{2}=-4 m_{s}\langle\bar{s} s\rangle+36 A \\
f^{0 \eta^{\prime}} f^{8 \eta^{\prime}} m_{\eta^{\prime}}^{2}+f^{0 \eta} f^{8 \eta} m_{\eta}^{2}=\frac{4}{\sqrt{3}} m_{s}\langle\bar{s} s\rangle \\
\left(f^{8 \eta}\right)^{2} m_{\eta}^{2}+\left(f^{8 \eta^{\prime}}\right)^{2} m_{\eta^{\prime}}^{2}=-\frac{4}{3} m_{s}\langle\bar{s} s\rangle
\end{gathered}
$$

Clearly, the two purely octet formulae can be used to find $f^{8 \eta}$ and $f^{8 \eta^{\prime}}$ if both $g_{\eta \gamma \gamma}$ and $g_{\eta^{\prime} \gamma \gamma}$ are known. The off-diagonal Dashen formula then expresses $f^{0 \eta}$ in terms of $f^{0 \eta^{\prime}}$. This leaves the two purely singlet formulae involving the stillundetermined decay constant $f^{0 \eta^{\prime}}$, the topological susceptibility coefficient $A$, and the coupling $g_{G \gamma \gamma}$. The advertised result follows immediately. If we neglect $g_{G \gamma \gamma}$, we can find $f^{0 \eta^{\prime}}$ from the singlet decay formula and thus determine $A$ from the remaining flavour singlet Dashen formula. This is the generalisation of the Witten-Veneziano formula.

Without neglecting $g_{G \gamma \gamma}$, the five equations give a self-consistent description of the radiative decays, but are non-predictive. It is therefore important to analyse more carefully whether it is really legitimate to neglect $g_{G \gamma \gamma}$. The argument is based on the fact that $g_{G \gamma \gamma}$ is both OZI suppressed and renormalisation group (RG) invariant [1]. Since violations of the OZI rule are associated with the $U_{A}(1)$ anomaly, it is a plausible conjecture that we can identify OZI-violating quantities by their dependence on the anomalous dimension associated with the non-trivial renormalisation of $J_{\mu 5}^{0}$ due to the anomaly. In this way, RG non-invariance can be used as a flag to indicate those quantities expected to show large OZI violations. If this conjecture is correct, then we would expect the OZI rule to be reasonably good for the RG invariant $g_{G \gamma \gamma}$, which would therefore be suppressed relative to $g_{\eta^{\prime} \gamma \gamma}$. (An important exception is of course the $\eta^{\prime}$ mass itself, which although obviously RG invariant is not zero in the chiral limit as it would be in the OZI limit of QCD.) Notice that this conjecture has been applied already with some success to the 'proton spin' problem in polarised deep inelastic scattering 沺. A related $1 / N_{c}$ discussion is given in [2].

\section{The Proof}

Consider first QCD by itself without the coupling to electromagnetism. The axial anomaly is

$\partial^{\mu} J_{\mu 5}^{a}=M_{a b} \phi_{5}^{a}+2 n_{f} Q \delta_{a 0}$

The notation is defined in ref[2]. The quark mass matrix is written as $m^{a} T^{a}$, so

$$
\left(\begin{array}{ccc}
m_{u} & 0 & 0 \\
0 & m_{d} & 0 \\
0 & 0 & m_{s}
\end{array}\right)=m^{0} \mathbf{1}+m^{3} T^{3}+m^{8} T^{8}
$$

The condensates are written as

$$
\left(\begin{array}{ccc}
\langle\bar{u} u\rangle & 0 & 0 \\
0 & \langle\bar{d} d\rangle & 0 \\
0 & 0 & \langle\bar{s} s\rangle
\end{array}\right)=\frac{1}{3}\left\langle\phi^{0}\right\rangle \mathbf{1}+2\left\langle\phi^{3}\right\rangle T^{3}+2\left\langle\phi^{8}\right\rangle T^{8}(12)
$$

where $\left\langle\phi^{c}\right\rangle$ is the $\operatorname{VEV}\left\langle\bar{q} T^{c} q\right\rangle$. Then

$M_{a b}=d_{a c b} m^{c}, \quad \Phi_{a b}=d_{a b c}\left\langle\phi^{c}\right\rangle$

The anomalous chiral Ward identities, at zero momentum, for the two-point Green functions of these operators are 2]

$$
\begin{gathered}
2 n_{f}\langle Q Q\rangle \delta_{a 0}+M_{a c}\left\langle\phi_{5}^{c} Q\right\rangle=0 \\
2 n_{f}\left\langle Q \phi_{5}^{b}\right\rangle \delta_{a 0}+M_{a c}\left\langle\phi_{5}^{c} \phi_{5}^{b}\right\rangle+\Phi_{a b}=0
\end{gathered}
$$

which imply

$M_{a c} M_{b d}\left\langle\phi_{5}^{c} \phi_{5}^{d}\right\rangle=-(M \Phi)_{a b}+\left(2 n_{f}\right)^{2}\langle Q Q\rangle \delta_{a 0} \delta_{b 0}(15)$

We also need the result for the general form of the topological susceptibility (see eq(6)):

$\chi(0) \equiv\langle Q Q\rangle=\frac{-A}{1-\left(2 n_{f}\right)^{2} A(M \Phi)_{00}^{-1}}$

Although the pseudoscalar operators $\phi_{5}^{a}$ and $Q$ indeed couple to the physical states $\eta^{\alpha}=\eta^{\prime}, \eta, \pi^{0}$, it is more convenient to redefine linear combinations such that the resulting propagator matrix is diagonal and properly normalised. So we define operators $\eta^{\alpha}$ and $G$ such that

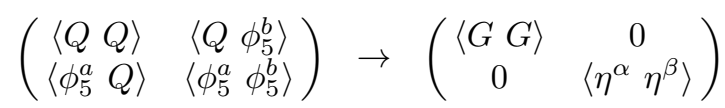

This is achieved by

$$
\begin{aligned}
G & =Q-\left\langle Q \phi_{5}^{a}\right\rangle\left(\left\langle\phi_{5} \phi_{5}\right\rangle\right)_{a b}^{-1} \phi_{5}^{b} \\
& =Q+2 n_{f} A \Phi_{0 b}^{-1} \phi_{5}^{b}
\end{aligned}
$$


and

$\eta^{\alpha}=f^{T \alpha a} \Phi_{a b}^{-1} \phi_{5}^{b}$

With this choice, the $\langle G G\rangle$ propagator is

$\langle G G\rangle=-A$

and we impose the normalisation

$\left\langle\eta^{\alpha} \eta^{\beta}\right\rangle=\frac{-1}{k^{2}-m_{\eta^{\alpha}}^{2}} \delta^{\alpha \beta}$

This implies that the constants $f^{a \alpha}$ in (19), which are simply the decay constants, must satisfy the (Dashen) identity

$$
\begin{aligned}
f^{a \alpha} m_{\alpha \beta}^{2} f^{T \beta b} & =\Phi_{a c}\left(\left\langle\phi_{5} \phi_{5}\right\rangle\right)_{c d}^{-1} \Phi_{d b} \\
& =-(M \Phi)_{a b}+\left(2 n_{f}\right)^{2} A \delta_{a 0} \delta_{b 0}
\end{aligned}
$$

The last line follows from the Ward identities (15) and (16) . In terms of these new operators, the anomaly equation (10) is:

$\partial^{\mu} J_{\mu 5}^{a}=f^{a \alpha} m_{\alpha \beta}^{2} \eta^{\beta}+2 n_{f} G \delta_{a 0}$

Now recall how conventional PCAC is applied to the calculation of $\pi^{0} \rightarrow \gamma \gamma$. The pion decay constant is defined as the coupling of the pion to the axial current

$$
\left\langle 0\left|J_{\mu 5}^{3}\right| \pi\right\rangle=i k_{\mu} f_{\pi} \Rightarrow\left\langle 0\left|\partial^{\mu} J_{\mu 5}^{3}\right| \pi\right\rangle=f_{\pi} m_{\pi}^{2}
$$

and satisfies the usual Dashen formula The next step is to define a 'phenomenological pion field' $\pi$ by

$\partial^{\mu} J_{\mu 5}^{3} \rightarrow f_{\pi} m_{\pi}^{2} \pi$

To include electromagnetism, the full anomaly equation is extended as in (3) to include the $F^{\mu \nu} \tilde{F}_{\mu \nu}$ contribution. Using (24) we have

$$
\begin{gathered}
\quad i k^{\mu}\left\langle\gamma \gamma\left|J_{\mu 5}^{3}\right| 0\right\rangle \\
=f_{\pi} m_{\pi}^{2}\langle\gamma \gamma|\pi| 0\rangle+a_{\mathrm{em}}^{a} \frac{\alpha}{8 \pi}\left\langle\gamma \gamma\left|F^{\mu \nu} \tilde{F}_{\mu \nu}\right| 0\right\rangle \\
=f_{\pi} m_{\pi}^{2}\langle\pi \pi\rangle\langle\gamma \gamma \mid \pi\rangle+a_{\mathrm{em}}^{a} \frac{\alpha}{8 \pi}\left\langle\gamma \gamma\left|F^{\mu \nu} \tilde{F}_{\mu \nu}\right| 0\right\rangle
\end{gathered}
$$

where $\langle\pi \pi\rangle$ is the pion propagator $-1 /\left(k^{2}-m_{\pi}^{2}\right)$. At zero momentum, the l.h.s. vanishes because of the explicit $k_{\mu}$ factor and the absence of massless poles. We therefore find,

$f_{\pi} g_{\pi \gamma \gamma}=a_{\mathrm{em}}^{3} \frac{\alpha}{\pi}$
In the full theory including the flavour singlet sector and the gluonic anomaly, we find a similar result. The 'phenomenological fields' are defined by (23) where the decay constants satisfy the generalised Dashen formula (22) . Notice, however, that they are not simply related to the couplings to the axial current as in (24) for the flavour nonsinglet. We therefore find:

$$
\begin{aligned}
& i k^{\mu}\left\langle\gamma \gamma\left|J_{\mu 5}^{a}\right| 0\right\rangle=f^{a \alpha} m_{\alpha \beta}^{2}\left\langle\gamma \gamma\left|\eta^{\beta}\right| 0\right\rangle \\
& +2 n_{f}\langle\gamma \gamma|G| 0\rangle \delta_{a 0}+a_{\mathrm{em}}^{a} \frac{\alpha}{8 \pi}\left\langle\gamma \gamma\left|F^{\mu \nu} \tilde{F}_{\mu \nu}\right| 0\right\rangle \\
& =f^{a \alpha} m_{\alpha \beta}^{2}\left\langle\eta^{\beta} \eta^{\gamma}\right\rangle\left\langle\gamma \gamma \mid \eta^{\gamma}\right\rangle \\
& +2 n_{f}\langle G G\rangle\langle\gamma \gamma \mid G\rangle \delta_{a 0}+a_{\mathrm{em}}^{a} \frac{\alpha}{8 \pi}\left\langle\gamma \gamma\left|F^{\mu \nu} \tilde{F}_{\mu \nu}\right| 0\right\rangle
\end{aligned}
$$

using the fact that the propagators are diagonal in the basis $\eta^{\alpha}, G$. Using the explicit expressions $(20),(21)$ for the Green functions, we find in this case:

$f^{a \alpha} g_{\eta^{a} \gamma \gamma}+2 n_{f} A g_{G \gamma \gamma} \delta_{a 0}=a_{\mathrm{em}}^{a} \frac{\alpha}{\pi}$

where the extra coupling $g_{G \gamma \gamma}$ is defined through (28). This completes the derivation. It is evidently a straightforward generalisation of conventional PCAC with the necessary modification of the usual formulae to take account of the extra gluonic contribution to the axial anomaly in the flavour singlet channel, the key point being the identification of the operators $\eta^{\alpha}$ and $G$ in (23).

Finally, notice that these methods may equally be applied to other decays involving the $\eta^{\prime}$ such as $\eta^{\prime} \rightarrow V \gamma$ where $V$ is a light $1^{-}$meson, $\eta^{\prime} \rightarrow \pi \pi \gamma$, $\psi \rightarrow \eta^{\prime} \gamma$, etc.

\section{Acknowledgements}

I would like to thank S. Narison and G. Veneziano for helpful discussions. This work was supported by the PPARC grant GR/L56374 and by the EC TMR grant FMRX-CT96-0008.

\section{REFERENCES}

1. G.M. Shore and G. Veneziano, Nucl. Phys. B381 (1992) 3.

2. G.M. Shore, hep-ph/9808217.

3. G.M. Shore, hep-ph/9812354.

4. G.M. Shore, hep-ph/9812355. 